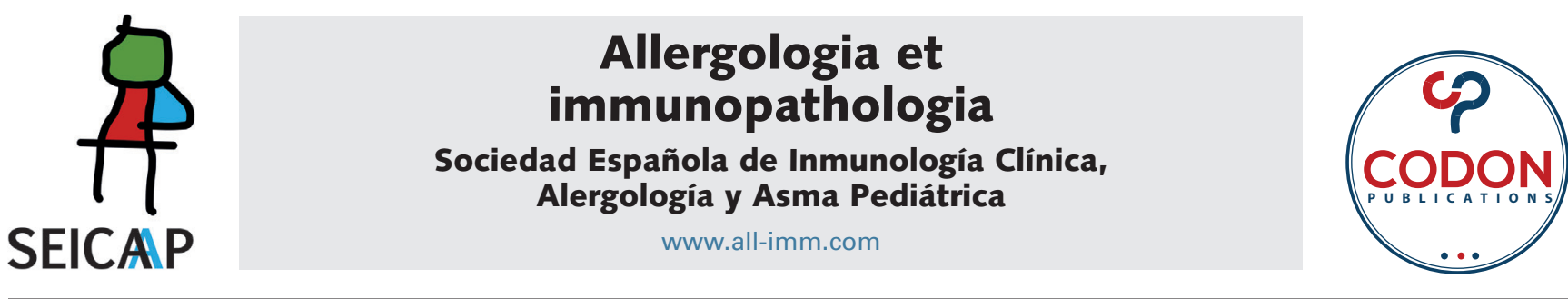

\title{
Effect of the SARS-CoV-2 pandemic on the control and severity of pediatric asthma
}

\author{
Silvia Sánchez-Garcíaa,b*, Javier Ruiz-Hornillos ${ }^{\mathrm{c}, \mathrm{d}, \mathrm{e}}$, Marta Bernaolaa, \\ Alicia Habernau-Mena ${ }^{f}$, Eva María Lasas, Javier Contrerash ${ }^{h}$ Rocío Candón-Morilloi, \\ Cristina Antón-Rodríguez ${ }^{\mathrm{e}}$, Carmelo Escudero ${ }^{\mathrm{a}, \mathrm{b}}$
}

\author{
${ }^{a}$ Department of Allergy, Hospital Infantil Universitario Niño Jesús, Madrid, Spain \\ ${ }^{b} A R A D y A L$ - RETICS Network RD16, Instituto de Salud Carlos III, IIS-P, FibHNJ, Madrid, Spain \\ 'Department of Allergy, Hospital Infanta Elena, Valdemoro, Madrid, Spain \\ UAM), Madrid, Spain \\ eFaculty of Medicine, Universidad Francisco de Vitoria, Madrid, Spain \\ fDepartment of Allergy, Complejo Hospitalario de Mérida, Badajoz, Spain \\ sPediatric Allergy Unit, Hospital Universitario Donostia, San Sebastián, Spain \\ 'Department of Allergy, La Paz University Hospital, Madrid, Spain \\ 'Department of Allergy, Hospital General La Mancha Centro, Alcázar de San Juan, Ciudad Real, Spain
}

IInstituto de Investigación Sanitaria-Fundación, Jiménez Díaz University Hospital, Universidad Autónoma de Madrid (IIS-FJD,

Received 23 August 2021; Accepted 29 October 2021

Available online 1 January 2022

\section{KEYWORDS}

asthma control;

asthma severity;

COVID;

pediatric asthma;

SARS-CoV-2

\begin{abstract}
Background: The novel disease caused by the new coronavirus SARS-CoV-2 has caused an unprecedented global pandemic. Care providers of asthmatic children are increasingly con-cerned; as viral infections are one of the primary triggers of asthma flare-up. However, the effect of SARS-CoV-2 as well as the generated worldwide lockdown on asthmatic children is unknown.

Objective: The aim of this study was to analyze the effects of pandemic SARS-CoV-2 in pediat-ric asthma control.

Material and Methods: A retrospective, open, transversal study was performed at five ter-tiary hospitals. Recruited patients were aged $<18$ years and had physician-diagnosed asthma. Information regarding the 2019 and 2020 seasons were provided.

Results: Data were collected from 107 children (age range: 3-18 years, mean age: 12 years). Well-controlled asthma was observed in 58 (54.2\%) patients in 2020 versus 30 (28\%) in 2019 , and $15(14 \%)$ patients had poorly controlled asthma in 2020 versus $28(26.2 \%)$ in 2019. In 2020, a decrease in exacerbations caused by allergies to pollen, dust mites, molds, and through other causes not related to SARS-CoV-2 infection was observed. An increase in exacerbations was observed due to animal dander, stress, physical exercise, and SARS-

CoV-2 infection. Children
\end{abstract}

*Corresponding author: Silvia Sanchez Garcia, Avda Menendez Pelayo 65, 28009-Madrid, Spain. Email address: silviasanchezgarcia@ hotmail.com 
had a reduced need for asthma-controlling medication, made fewer visits to healthcare providers and had lesser need of treatment with oral corticosteroids if compared with the same season of 2019.

Conclusion: Pediatric asthma control improved, the need for controller medication declined, and fewer visits to healthcare providers were made during the pandemic if compared with the 2019 season.

(c) 2022 Codon Publications. Published by Codon Publications.

\section{Introduction}

The novel disease caused by the new coronavirus SARSCoV-2 has caused an unprecedented global pandemic, ${ }^{1}$ affecting not only health systems but social and economic systems as well. The lack of knowledge of the virus' behavior, its impact on chronic respiratory patients, clinical characteristics of the disease, and possible comorbidities have generated chaos and alarm among healthcare providers and the general population. Care providers of asthmatic children are increasingly concerned, as asthma is the most common chronic respiratory disease at this age and is responsible for morbidity and decreased quality of life. ${ }^{1}$ Viral infections are one of the primary triggers of asthma flare-up. ${ }^{1}$ The few reports to date are conflicting as Ibrahim et al. reported a SARS-CoV-2 positivity rate among children who attended the emergency room (ER) as low as $0.9 \%,{ }^{2}$ while other authors have published a higher percentage (up to $31.3 \%),{ }^{3}$ revealing the considerable lack of knowledge about the disease and its risk factors. Viral infections are known triggers of asthma flare-ups, especially in asthmatic children. Authorities have recognized moderate and severe asthma as risk factors of severe SARS-CoV-2 infection, but evidence is scarce regarding COVID-19 (coronavirus disease emerged in 2019 in Wuhan [China]) as a trigger for exacerbated asthma. The first studies, from direct clinical observations, seem to confirm that children are not a highrisk population, nor are asthmatics particularly vulnerable to infection by SARS-CoV- $2 .{ }^{5}$ However, these studies are few, performed with haste because of the limitations of the moment, and many are based on expert opinions, rather than clinical data. The onset of the pandemic generated a worldwide lockdown, including in Spain, forcing children to stay inside their homes. Access to both primary and specialized care has been limited due to higher proportion of COVID-19 patients, parental fear of visiting any healthcare facility, and emergency departments being burdened, thereby restricting considerably the regular attendance of asthmatic patients and a decline in lung function tests. The effects of these factors on asthma control are unknown. Although clinical observation has demonstrated neither a higher risk of asthma flare-ups caused by COVID-19 among children nor a higher prevalence of COVID-19 in asthmatic population, ${ }^{4,5}$ the overlapping symptoms of the two conditions must be considered. The increased stress and anxiety experienced by patients ${ }^{6}$ overlapped with both asthma and COVID-19 symptoms and may lead to misunderstanding. This difficulty in obtaining a correct diagnosis among the three entities could lead to asthmatic symptoms being over- or underestimated. Existing publications about the effects of previously described factors are scarce and based on limited clinical observations, consensus documents, and expert reviews. ${ }^{7,8}$ The aim of this study was to analyze the influence of COVID-19 pandemic in pediatric asthma control.

\section{Material and Methods}

\section{Objective}

The primary objective was to discover how well-controlled the asthma of pediatric patients was during the pandemic lockdown period, from mid-March to mid-June 2020. We examined both the direct influence of SARS-CoV-2 infection and the indirect effects of lockdown and state of emergency in healthcare systems. Secondary objectives were to (1) determine the SARS-CoV-2 infection rate in asthmatic patients aged less than 18 years, and (2) compare the clinical and epidemiological characteristics of children who experienced COVID-19 illness with those not having COVID-19 infection.

\section{Study design and recruitment}

A retrospective, open, multicenter, transversal study was performed at five tertiary hospitals in Spain. The study was approved by the Medical Ethics Committee of Niño Jesús University Children's Hospital, Madrid, Spain, Code: R-0055/20, and classified by the Spanish Agency for Medicines and Health Products with Code SEA-COR-2020-09. The study was carried out in accordance with the principles stated in the Declaration of Helsinki. Informed consent was obtained from all parents/guardians.

Participants were consecutively recruited from the patients attending the allergy clinic. The inclusion criteria were: age $<18$ years, a physician's diagnosis of asthma, and residence in Spain from March 15, 2020 to June 15, 2020. Each patient was invited to participate at the time of their regular visit to the clinic for asthma control. Investigators entered the collected data into online database for data protection. Data were anonymized before being added to the database. The data included maintenance treatment, asthma control, admittance to hospital/emergency department, visits to primary care, nonscheduled visits to an asthma specialist, and number and causes of exacerbations during 2019 and 2020 seasons. Information not recalled was gathered from computerized medical records. Informed consent was obtained from all subjects, parents, and guardians.

The degree of asthma control was measured according to the 2020 Global Initiative for Asthma (GINA) guidelines. ${ }^{9}$ 


\section{Statistical analysis}

Data analysis was performed using the SPSS version 21 software. Descriptive analyses were performed using mean values and standard deviations for quantitative variables and proportional (percentage) distributions were used for qualitative ones. Differences between 2019 and 2020 were evaluated with cross tables and the Chi-square test. Variables compared were population habitual housing, maintenance treatment for asthma, degree of asthma control, primary care or specialist review required, corticosteroid or biological treatment required, and possible exacerbation triggers. Differences with $\mathrm{P} \leq 0.05$ were considered statistically significant.

\section{Results}

\section{Demographic data}

Data were collected from 107 children, including 73 (68.2\%) males. Their age ranged from 3 to 18 years, with a mean age of 12 years. The mean period of evolution of the disease since asthma diagnosis was 4.9 years (1 month-12 years) (Table 1).

\section{Asthma control and severity during the COVID-19 pandemic}

Every item evaluated to estimate asthma control according to GINA ${ }^{9}$ improved during March-June 2020, compared with the same period of 2019, with significant improvements in daily symptoms and activity limitation $(P<0.01)$ (See Figure 1A: Characteristics of asthma control).

Table 1 Characteristics of included asthma patients.

\begin{tabular}{lc}
\hline Demographic characteristics & \\
\hline Age (years) (mean, SD) & $12.1(3.0)$ \\
Gender, N (\%) & $73(68.2 \%)$ \\
Male & $34(31.8 \%)$ \\
Female & $4.9(3.1)$ \\
Time from asthma diagnosis (years) (mean, SD) & \\
Number of inhabitants at place of residence, N (\%) & $6(5.6 \%)$ \\
$<5000$ & $63(58.9 \%)$ \\
$5000-100,000$ & $6(5.6 \%)$ \\
$100,001-500,000$ & $32(29.9 \%)$ \\
$>500,000$ & \\
\hline Asthma characteristics prior to pandemic & \\
Asthma severity N (\%) & $50(46.7 \%)$ \\
\hline Mild & $21(19.6 \%)$ \\
Moderate & $36(33.6 \%)$ \\
\hline Severe & \\
\hline Asthma control N (\%) & $30(28 \%)$ \\
\hline Well controlled & $49(45.8 \%)$ \\
Partially controlled & $28(26.2 \%)$ \\
\hline Poorly controlled & \\
\hline
\end{tabular}

SD, standard deviation.
(A)

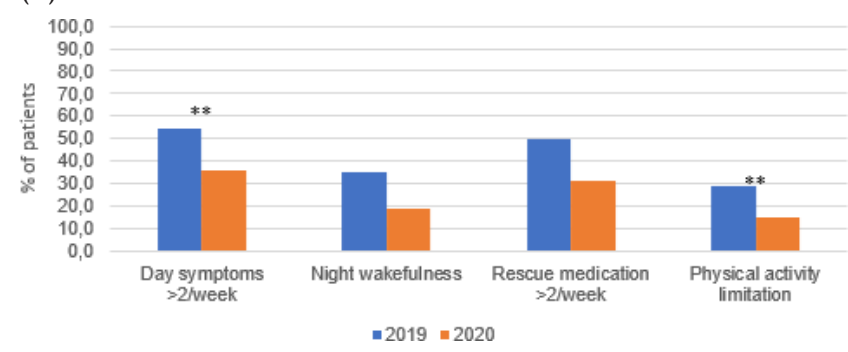

(B)

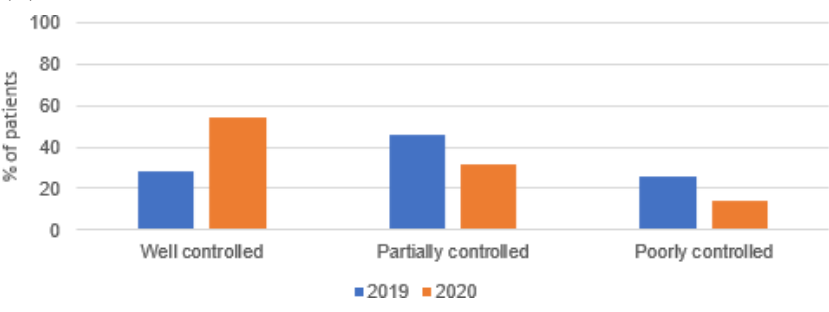

(C)

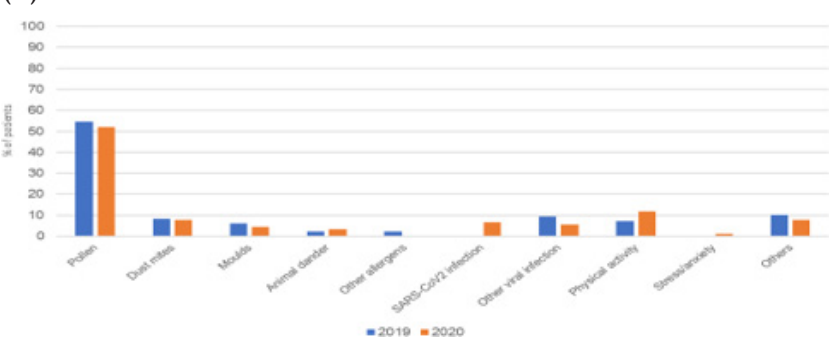

(D)

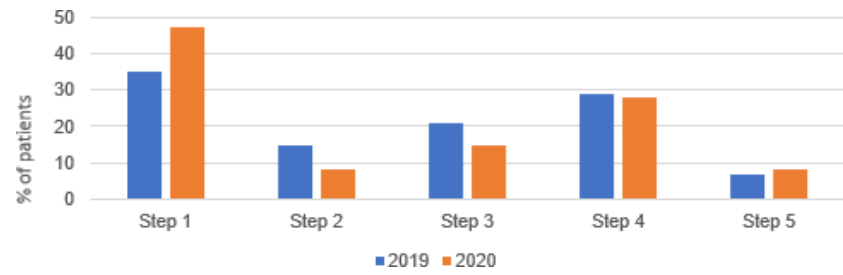

Figure 1 (A) Characteristics of asthma control; (B) Level of asthma control; (C) Triggers of asthma exacerbation; (D) Number of patients per step of controller medication according to GINA 2020.

During the 2020 pandemic, 58 (54.2\%) patients had well-controlled asthma compared with 30 (28\%) in 2019, while 15 (14\%) patients in 2020 had poorly controlled asthma compared with 28 (26.2\%) in 2019. However, not statistically significant between-year differences were determined (See Figure 1B: Level of asthma control).

A total of 97 exacerbations occurred during March-June 2019 whereas 92 happened during the same period in 2020. Compared with 2019, in 2020 there was a decrease in the frequency of exacerbations caused by pollen allergy (48 [52.2\%] vs. 53 [54.6\%]), dust mites (7 [7.6\%] vs. 8 [8.2\%]), molds (4 [4.3\%] vs. 6 [6.2\%]), and non-SARS-CoV-2 infections (5 [5.4\%] vs. 9 [9.3\%]). However, an increase in frequency was observed for cases of animal dander (3 [3.3\%] vs. 2 [2.1\%]), stress (1 [1.1\%] vs. 0), physical exercise (11 [12\%] vs. 7 [7.2\%]), and SARS-CoV-2 infection (6 [6.5\%] vs. 0). No statistically significant differences were detected (Figure 1C: Triggers of asthma exacerbation). 
In 2020, children had less need for asthma-controlling medication and were, therefore, at a lower therapeutic step according to GINA 2020 (Figure 1D: Number of patients per step of controller medication according to GINA 2020). Regarding asthma severity, comparing 2020 with 2019, more children had mild asthma (55 [51.4\%] vs. 50 [46.7\%]; $\mathrm{P}<0.01)$, and there were fewer patients with moderate asthma (15 [14\%] vs. 21 [19.3\%]; $\mathrm{P}<0.01)$.

During the spring of 2020, decrease in visits to emergency department, primary healthcare centers, nonscheduled visits to specialists, and the need for oral corticosteroid treatment were observed when compared with the same period of 2019. Only one patient was admitted to hospital in 2019 due to asthma attack but none in 2020.

\section{SARS-CoV-2 infection in pediatric asthmatic patients}

Serological testing (IgG) for SARS-CoV-2 was performed in 21 of the 107 (19.6\%) patients. The remaining subjects' parents did not consent to providing the sample. Only two patients (1.9\%) were diagnosed with COVID-19 through serological testing or physician's diagnosis. None of these children was observed to have less asthma control in comparison to uninfected children. No patient was hospitalized due to COVID-19.

\section{DISCUSSION}

The pandemic caused by the respiratory virus SARS-CoV-2 has provoked a chaotic situation globally, with many remaining unknown. ${ }^{2}$ Special concern is dedicated to chronic respiratory patients, such as pediatric asthmatics. Although corona viruses are a relatively common trigger for asthma flare-ups, data related to the new SARS-CoV-2 have elicited controversy. Some authors have reported a decrease in asthma attacks, while others have observed a worsening situation in asthma control. ${ }^{10}$ To our knowledge, this was the first multicenter study to confirm better pediatric asthma control, a decrease in the need for controller medication, and a fewer visits to emergency departments and primary healthcare centers during the pandemic if compared with the 2019 season. These results were consistent with the results of the only single-center report published to date in our country. ${ }^{5}$

A decline in hospital admissions for asthma exacerbations was reported during the pandemic. ${ }^{11-13}$ We observed a decrease from $12 \%$ of patients being admitted to emergency departments during 2019 to $2.8 \%$ in 2020, a change similar to that observed in other reports. ${ }^{11,13}$ Chavasse reported a reduction of over $90 \%$ in hospital admissions in London caused by asthma flare-ups; however, this could be related to different selection criteria. ${ }^{12}$ While the mentioned study analyzed an emergency department serving general population, our data were collected from specialist clinicians whose patients have been carefully treated and followed in allergy departments for over the years.

Country-specific differences in virus control measures and access to healthcare could also have affected the observed differences in hospitalization. In fact, we observed fewer visits to primary healthcare centers during 2020. This could be related to the closure of a percentage of healthcare facilities as their medical staff were transferred to COVID-19 attendance, as confirmed by one of our patients. However, the decrease in need for treatment with corticosteroids may confirm a real decrease in asthma flare-ups, rather than solely the fear of visiting any healthcare facility or limited access to the same. There was a notable significant improvement in asthma control, especially in relation to daily symptoms and limits to physical exercise. During lockdown, children did not feel affected by their asthma. Decrease in daily practice of sports could be one possible reason.

One of the most reported triggers of asthma exacerbation was physical exercise, primarily during the first few days after lockdown. The most probable reason for this could be a failure of routine training, rather than a real asthma attack, even when exacerbated by a pollen peak period during spring.

Although there was no statistically significant difference, exacerbations because of pet allergies increased, presumably due to increased indoor exposures. Regarding exposure to other environmental allergens, although significant differences were not observed, the lockdown exposed the patients to indoor allergens for a longer period. There was an increase in exacerbations that were probably due to this factor. Accordingly, we observed fewer exacerbations caused by infections when compared with 2019. Similarly, Gajaweera et al. reported a case series of 58 patients, in which $48 \%$ of the children self-reported better control of asthma than they had during the same period in the previous year. ${ }^{14}$ The primary reasons were fewer infections and less exposure to pollen due to lockdown.

Regarding severity, more patients had mild asthma attack during the pandemic whereas in 2019, there was more moderate disease. This could be related to the decline in asthma exacerbations and lack of infections, less exposure to pollen, and low physical exercise. Increase in telephone consultations with specialists could be related to significant increase in using telemedicine during the pandemic among pediatric asthma patients, revealing more a manner of consultancy, rather than a real need of a nonscheduled visit. ${ }^{15}$

Recent studies have not observed increased vulnerability to worst asthma, ${ }^{5}$ or a significant relation between asthma and COVID-19.9 The primary limitations of our study are that the data were collected retrospectively, and that physician-diagnosed COVID-19 was confirmed in only two cases. The state of infection in the remaining children was unknown, as their parents refused to consent to serological testing. The strengths of this report are the multicenter enrolment of patients and their different patterns of allergen and infection exposure. Moreover, the data were collected by a trained allergist and surveys were avoided.

\section{CONCLUSION}

Pediatric asthmatic patients resulted in a better control of the disease during the lockdown period. This report reveals the relevance of avoiding allergens in pediatric 
asthma, as the lockdown demonstrated to improve control over asthma. Another possible cause of this improvement could be the decrease in other viral infections because of social distancing.

\section{Acknowledgments}

Authors thank AstraZeneca for financial support.

\section{Conflict of interest}

The authors declare no potential conflicts of interest regarding research, authorship, and/or publication of this article.

\section{References}

1. Papadopoulos NG, Arakawa H, Carlsen KH, Gern J, Lemanske R, Le Souef $P$, et al. International consensus on (ICON) pediatric asthma. Allergy. 2012;67(8):976-97. https://doi.org/10.1111/j. 1398-2049995.2012.02865.x

2. Ibrahim LF, Tosif S, McNab S, Hall S, Lee HJ, Lewena S, et al. SARS-CoV-2 testing and outcomes in the first 30 days after the first case of COVID-19 at an Australian children's hospital. Emerg Med Australas. 2020;32(5):801-8. https://doi.org/ 10.1111/1742-6723.13550

3. Chao JY, Derespina KR, Herold BC, Goldman DL, Aldrich M, Weingarten J, et al. Clinical characteristics and outcomes of hospitalized and critically ill children and adolescents with coronavirus disease 2019 at a tertiary care medical center in New York City. J Pediatr. 2020;223:14-19.e2. https://doi.org/ 10.1016/j.jpeds.2020.05.006

4. Licari A, Votto M, Brambilla I, Castagnoli R, Piccotti E, Olcese R, et al. Allergy and asthma in children and adolescents during the COVID outbreak: What we know and how we could prevent allergy and asthma flares. Allergy. 2020;75(9):2402-5. https:// doi.org/10.1111/all.14369

5. Ruano FJ, Somoza Álvarez ML, Haroun-Díaz E, Vazquez de la Torre M, Lopez-Gonzalez P, Prieto-Moreno A, et al. Impact of the COVID-19 pandemic in children with allergic asthma. J Allergy Clin Immunol Pract. 2020;8(9):3172-4.e1. https://doi. org/10.1016/j.jaip.2020.07.019

6. Gonzalez-Diaz SN, Martin B, Villarreal-Gonzalez RV, de LiraQuezada CE, Macouzet-Sanchez C, Macias-Weinmann A, et al. Psychological impact of the COVID-19 pandemic on patients with allergic diseases. World Allergy Organ J. 2021;14(3):100510. https://doi.org/10.1016/j.waojou.2021.100510

7. Levin M, Ansotegui IJ, Bernstein J, Chang YS, Chikhladze M, Ebisawa $M$, et al. Acute asthma management during SARSCoV2 pandemic 2020. World Allergy Organ J. 2020 May 14;13(5):100125. https://doi.org/10.1016/j.waojou.2020.100125

8. Scadding GK, Hellings PW, Bachert C, Bjermer L, Diamant Z, Gevaert $P$, et al. Allergic respiratory disease care in the COVID19 era: A EUFOREA statement. World Allergy Organ J. 2020 May 16;13(5):100124. https://doi.org/10.1016/j.waojou.2020.100124

9. GINA: Global Strategy for Asthma Management and Prevention. 2020 GINA guidelines [Internet]. Available from: https://ginasthma.org/ (Date of citation: May 2021).

10. Papadopoulos NG, Mathioudakis AG, Custovic A, Deschildre A, Phipatanakul W, Wong G, et al. Childhood asthma outcomes during the COVID-19 pandemic: Findings from the PeARL multinational cohort. Allergy. 2021. (Published online ahead of print, 2021 Feb 20). https://doi.org/10.1111/all.14787

11. Krivec U, KofolSeliger A, Tursic J. COVID-19 lockdown dropped the rate of paediatric asthma admissions. Arch Dis Child. 2020;105(8):809-10. https://doi.org/10.1136/archdischild-2020319522

12. Chavasse RJ. Covid-19: Reduced asthma presentations in children. BMJ. 2020 Jul 15;370:m2806. https://doi.org/10.1136/ bmj.m2806

13. Ciprandi G, Licari A, Filippelli G, Tosca MA, Marseglia GL. Children and adolescents with allergy and/or asthma seem to be protected from coronavirus disease 2019. Ann Allergy Asthma Immunol. 2020;125(3):361-2. https://doi.org/10.1016/j. anai.2020.06.001

14. Gajaweera H, Oladele D, Connett G. Evaluation of the impact of shielding to avoid COVID-19 infection on respiratory symptoms in children with severe asthma. Arch Dis Child. 2021;106(4):e23. https://doi.org/10.1136/archdischild-2020-320498

15. Sánchez-García S, Ruiz-Hornillos J, Escudero C. Pediatric asthma management during the COVID-19 pandemic: Results of a national survey. Pediatr Allergy Immunol Pulmonol. 2020;33:199-203. https://doi.org/10.1089/ped.2020.1250 